\title{
Mystic Reflection Groups ${ }^{\star}$
}

Yuri BAZLOV ${ }^{\dagger}$ and Arkady BERENSTEIN ${ }^{\ddagger}$

$\dagger$ School of Mathematics, University of Manchester, Oxford Road, Manchester, M13 9PL, UK

E-mail: yuri.bazlov@manchester.ac.uk

$\ddagger$ Department of Mathematics, University of Oregon, Eugene, OR 97403, USA

E-mail: arkadiy@math.uoregon.edu

Received December 25, 2013, in final form March 24, 2014; Published online April 04, 2014

http://dx.doi.org/10.3842/SIGMA.2014.040

\begin{abstract}
This paper aims to systematically study mystic reflection groups that emerged independently in the paper [Selecta Math. (N.S.) 14 (2009), 325-372] by the authors and in the paper [Algebr. Represent. Theory 13 (2010), 127-158] by Kirkman, Kuzmanovich and Zhang. A detailed analysis of this class of groups reveals that they are in a nontrivial correspondence with the complex reflection groups $G(m, p, n)$. We also prove that the group algebras of corresponding groups are isomorphic and classify all such groups up to isomorphism.
\end{abstract}

Key words: complex reflection; mystic reflection group; thick subgroups

2010 Mathematics Subject Classification: 16G99; 20F55; 16S80

\section{Introduction}

Let $V$ be a complex vector space with basis $\left\{x_{1}, \ldots, x_{n}\right\}$. Denote by $S(V)$ the symmetric algebra of $V$. It is a fundamental fact that the algebra $S(V)^{\mathbb{S}_{n}}$ of symmetric polynomials is isomorphic to $S(V)$. More generally, the Chevalley-Shephard-Todd theorem asserts that for a finite group $G \subset \mathrm{GL}(V), S(V)^{G}$ is isomorphic to $S(V)$ if and only if $G$ is generated by complex reflections on $V$.

In a remarkable paper [2], Kirkman, Kuzmanovich and Zhang solved the following problem:

Problem 1.1. Given a complex matrix $\mathbf{q}=\left(q_{i j}, 1 \leq i, j \leq n\right)$ with $q_{i j} q_{j i}=1, q_{i i}=1$, let $S_{\mathbf{q}}(V)$ be the algebra generated by $V$ subject to the relations $x_{i} x_{j}=q_{i j} x_{j} x_{i}$ for $1 \leq i, j \leq n$. Find all finite groups $G$ such that

(a) G acts on the algebra $S_{\mathbf{q}}(V)$ by degree-preserving automorphisms;

(b) The fixed point algebra $S_{\mathbf{q}}(V)^{G}$ is isomorphic to $S_{\mathbf{q}^{\prime}}(V)$ for some $\mathbf{q}^{\prime}$.

We will refer to a group $G$ satisfying (a) and (b) above as a mystic reflection group. Independently, in [1] we solved the following problem:

Problem 1.2. Classify all algebras $\mathcal{A}$ such that

- $\mathcal{A}$ is generated by the space $V$, its dual $V^{*}$ and a finite group $G \subset \operatorname{GL}(V)$;

- $\mathcal{A}$ admits a triangular decomposition $\mathcal{A}=S_{\mathbf{q}}(V) \otimes \mathbb{C} G \otimes S_{\mathbf{q}}\left(V^{*}\right)$ for some $\mathbf{q}$ as above;

- $S_{\mathbf{q}}(V)$ and $S_{\mathbf{q}}\left(V^{*}\right)$ are invariant under conjugation by elements of $G$, and the restriction of these conjugation representations to $V$ and $V^{*}$ is the natural action of $G$ on these spaces;

- $y_{j} x_{i}-q_{i j} x_{i} y_{j} \in \mathbb{C} G$ for all $i, j$, where $\left\{y_{1}, \ldots, y_{n}\right\}$ is the basis of $V^{*}$ dual to $\left\{x_{1}, \ldots, x_{n}\right\}$.

${ }^{\star}$ This paper is a contribution to the Special Issue in honor of Anatol Kirillov and Tetsuji Miwa. The full collection is available at http://www.emis.de/journals/SIGMA/InfiniteAnalysis2013.html 
Comparing [2, Theorem 1.1 and Corollary 5.6] and [1, Theorem 0.7], one obtains the following surprising result:

Theorem 1.3. A group $G$ solves Problem 1.2 if and only if it is a mystic reflection group.

The goal of this paper is to "demystify" the mystic reflection groups, develop their structural theory, show that their group algebras are isomorphic to those of complex reflection groups, and to deduce Problem 1.1 directly from the classical Chevalley-Shepard-Todd theorem.

\section{Main results}

We start with a new notion of "mystical equivalence" of group actions, which is crucial for what follows.

Definition 2.1. Let $\triangleright: G \times \mathcal{V} \rightarrow \mathcal{V}, \triangleright^{\prime}: G^{\prime} \times \mathcal{V} \rightarrow \mathcal{V}$ be faithful actions of finite groups $G$, respectively $G^{\prime}$, on a complex vector space $\mathcal{V}$. We say that the actions $\triangleright$ and $\triangleright^{\prime}$ are mystically equivalent, if

$$
\rho\left(e_{G}\right)=\rho^{\prime}\left(e_{G^{\prime}}\right),
$$

where $\rho: \mathbb{C} G \rightarrow \operatorname{End}_{\mathbb{C}} \mathcal{V}$ and $\rho^{\prime}: \mathbb{C} G^{\prime} \rightarrow \operatorname{End}_{\mathbb{C}} \mathcal{V}$ are the algebra homomorphisms defined by the actions, and $e_{G}$ denotes the element $\sum_{g \in G} g$ of $\mathbb{C} G$.

Mystical equivalence of the actions of $G$ and $G^{\prime}$ is a strengthening of the condition that the respective spaces $\mathcal{V}^{G}$ and $\mathcal{V}^{G^{\prime}}$ of invariants are equal, due to the following obvious result.

Lemma 2.2. If a $G$-action and a $G^{\prime}$-action on $\mathcal{V}$ are mystically equivalent, then $\mathcal{V}^{G}=\mathcal{V}^{G^{\prime}}$.

We will use the lemma in the situation where $\mathcal{V}=S(V)$ where $V$ is a vector space over $\mathbb{C}$ with a chosen basis $\left\{x_{1}, \ldots, x_{n}\right\}$. Throughout the paper, $n \geq 2$. Denote by $\mathbb{G}_{n}$ the group of monomial matrices on $V$, that is, matrices in $\mathrm{GL}_{n}(\mathbb{C})$ with exactly $n$ non-zero entries. In other words,

$$
\mathbb{G}_{n}=\left(\mathbb{C}^{\times}\right)^{n} \rtimes \mathbb{S}_{n} .
$$

Here $\left(\mathbb{C}^{\times}\right)^{n}$ is naturally identified with the group of diagonal matrices in $\mathrm{GL}_{n}(\mathbb{C})$ and acts on $V$ by scaling the basis $\left\{x_{1}, \ldots, x_{n}\right\}$. The symmetric group $\mathbb{S}_{n}$ is identified with the group of permutation matrices and acts on $V$ by permuting the same basis. Note that $\mathbb{G}_{n}$ is the normalizer of the torus $\left(\mathbb{C}^{\times}\right)^{n}$ in $\mathrm{GL}_{n}(\mathbb{C})$. In particular, $\mathbb{S}_{n}$ acts on $\left(\mathbb{C}^{\times}\right)^{n}$ by conjugation. When we write $t w \in \mathbb{G}_{n}$, we will imply that $t \in\left(\mathbb{C}^{\times}\right)^{n}$ and $w \in \mathbb{S}_{n}$; every element of $\mathbb{G}_{n}$ can be uniquely written in this way.

Clearly, $\mathbb{G}_{n}$ is generated by $s_{1}, \ldots, s_{n-1}$ and $t_{j}^{(\zeta)}, 1 \leq j \leq n, \zeta \in \mathbb{C}^{\times}$, where

- $s_{i} \in \mathbb{S}_{n}$ is the permutation of $\left\{x_{1}, \ldots, x_{n}\right\}$ which swaps $x_{i}$ and $x_{i+1}$;

- $t_{j}^{(\zeta)} \in\left(\mathbb{C}^{\times}\right)^{n} \operatorname{maps} x_{k}$ to $\zeta^{\delta_{j k}} x_{k}$.

We find it very convenient to use the linear character

$\operatorname{det}: \mathbb{G}_{n} \rightarrow \mathbb{C}^{\times}$

of $\mathbb{G}_{n}$, which is just the restriction of the determinant character of $\mathrm{GL}_{n}(\mathbb{C})$ to $\mathbb{G}_{n}$. In particular,

$$
\operatorname{det} s_{i}=-1, \quad \operatorname{det} w \in\{ \pm 1\} \text { for } w \in \mathbb{S}_{n}, \quad \operatorname{det}\left(t_{1}^{\left(\zeta_{1}\right)} t_{2}^{\left(\zeta_{2}\right)} \cdots t_{n}^{\left(\zeta_{n}\right)}\right)=\zeta_{1} \zeta_{2} \cdots \zeta_{n} .
$$

Next, we introduce two different faithful actions of $\mathbb{G}_{n}$ on $S(V)$ using the natural basis $\left\{x_{1}^{k_{1}} \cdots x_{n}^{k_{n}}: k_{1}, \ldots, k_{n} \in \mathbb{Z}_{\geq 0}\right\}$ of $S(V)$. (At the moment, we are not using any multiplication on $S(V)$.) 


\section{Proposition 2.3.}

(a) There exist (unique) faithful actions $\triangleright_{+}, \triangleright_{-}$of $\mathbb{G}_{n}$ on $S(V)$ such that

$$
\begin{aligned}
& s_{i} \triangleright_{ \pm} x_{1}^{k_{1}} \cdots x_{n}^{k_{n}}=( \pm 1)^{k_{i} k_{i+1}} x_{1}^{k_{1}} \cdots x_{i}^{k_{i+1}} x_{i+1}^{k_{i}} \cdots x_{n}^{k_{n}}, \\
& t_{j}^{(\zeta)} \triangleright_{ \pm} x_{1}^{k_{1}} \cdots x_{n}^{k_{n}}=\zeta^{k_{j}} x_{1}^{k_{1}} \cdots x_{n}^{k_{n}}
\end{aligned}
$$

for any $i=1, \ldots, n-1, j=1, \ldots, n, \zeta \in \mathbb{C}^{\times}$. Both actions extend the defining action of $\mathbb{G}_{n}$ on $V$.

(b) The action $\triangleright_{+}$of $\mathbb{G}_{n}$ on $S(V)$ is compatible with the natural commutative multiplication on $S(V)$, in the sense that $\mathbb{G}_{n}$ acts by automorphisms of the algebra $S(V)$.

(c) The action $\triangleright_{-}$of $\mathbb{G}_{n}$ on $S(V)$ is compatible with the algebra structure $S_{-\mathbf{1}}(V)$ on $S(V)$ (which is $S_{\mathbf{q}}(V)$ with $q_{i j}=-1$ for all $i \neq j$ );

(d) $\rho_{+}\left(\mathbb{C}_{n}\right)=\rho_{-}\left(\mathbb{C}_{n}\right)$, where $\rho_{ \pm}: \mathbb{C}_{n} \rightarrow \operatorname{End}_{\mathbb{C}} S(V)$ are the algebra homomorphisms arising from the actions $\triangleright_{ \pm}$. Moreover, $\rho_{-}=\rho_{+} \circ J$ for some algebra automorphism $J$ of $\mathbb{C} \mathbb{G}_{n}$.

Remark 2.4. By a powerful result on group actions on integral domains, see Corollary 4.2 from the Appendix taken with $R=\mathbb{C}, A=S(V)$, the action $\triangleright_{+}$is faithful, and moreover the corresponding algebra homomorphism $\rho_{+}: \mathbb{C}_{n} \rightarrow \operatorname{End}_{\mathbb{C}} S(V)$ is injective. It follows from (d) that $\rho_{-}: \mathbb{C G}_{n} \rightarrow \operatorname{End}_{\mathbb{C}} S_{-\mathbf{1}}(V)$ is also injective. This fact does not readily follow from classical results.

At this point, we restrict our attention to finite subgroups $G$ of $\mathbb{G}_{n}$ - namely, to ShephardTodd's imprimitive complex reflection groups $G(m, p, n)$ and the groups $W_{\mathcal{C}, \mathcal{C}^{\prime}}$, introduced independently in [1] and [2] and defined as follows. Let $n \geq 1$ and $\mathcal{C}^{\prime} \subseteq \mathcal{C}$ be two finite subgroups of $\mathbb{C}^{\times}$of orders $\frac{m}{p}, m$ respectively. Then

$$
\begin{aligned}
& G(m, p, n)=\left\{t w \in \mathcal{C}^{n} \rtimes \mathbb{S}_{n}: \operatorname{det} t \in \mathcal{C}^{\prime}\right\}, \\
& W_{\mathcal{C}, \mathcal{C}^{\prime}}=\left\{t w \in \mathcal{C}^{n} \rtimes \mathbb{S}_{n}: \operatorname{det}(t w) \in \mathcal{C}^{\prime}\right\} .
\end{aligned}
$$

The similarity of the two definitions manifests itself in our first main result where a correspondence, $\mu$, between the two classes of subgroups of $\mathbb{G}_{n}$ is established.

Main Theorem 2.5. Given $n \geq 1$, an even $m \geq 2$ and a divisor $p$ of $m$, let $G=G(m, p, n)$. Then there exists a unique finite subgroup $\mu(G) \subset \mathbb{G}_{n}$ such that the restriction of $\triangleright_{-}$onto $\mu(G)$ is mystically equivalent to $\triangleright_{+}$on $G$. In fact,

$$
\mu(G)=W_{\mathcal{C}, \mathcal{C}^{\prime}},
$$

where $|\mathcal{C}|=m,\left|\mathcal{C}^{\prime}\right|=\frac{m}{p}$.

The definition of the group $\mu(G)$ suggests that the invariants of $\mu(G(m, p, n))=W_{\mathcal{C}, \mathcal{C}^{\prime}}$ should be viewed in the noncommutative algebra $S_{-\mathbf{1}}(V)$, where this group acts via $\triangleright_{-}$. To describe these invariants, introduce the following elements in the space $S(V)$ :

$$
p_{k}^{(m)}=\sum_{i=1}^{n} x_{i}^{k m}, \quad k=1, \ldots, n-1, \quad r^{(l)}=x_{1}^{l} x_{2}^{l} \cdots x_{n}^{l} .
$$

The classical result of Shephard-Todd and Chevalley asserts that the subalgebra $S(V)^{G(m, p, n)}$ of $S(V)$ (with respect to the natural commutative product on $S(V)$ ) equals the polynomial algebra $\mathbb{C}\left[p_{1}^{(m)}, \ldots, p_{n-1}^{(m)}, r^{\left(\frac{m}{p}\right)}\right]$.

Our mystic equivalence construction immediately leads to the following result, first obtained by Kirkman, Kuzmanovich and Zhang as a key ingredient in the classification theorem [2, Theorem 1.1]. 
Theorem 2.6. In the notation of Theorem 2.5, let $m$ be even. Then in the algebra $S_{-\mathbf{1}}(V)$,

(a) the elements $p_{1}^{(|\mathcal{C}|)}, \ldots, p_{n-1}^{(|\mathcal{C}|)}, r^{\left(\left|\mathcal{C}^{\prime}\right|\right)}$ are pairwise commuting invariants of the group $W_{\mathcal{C}, \mathcal{C}^{\prime}}$;

(b) $S_{-\mathbf{1}}(V)^{W_{\mathcal{C}, \mathcal{C}^{\prime}}}=\mathbb{C}\left[p_{1}^{(|\mathcal{C}|)}, \ldots, p_{n-1}^{(|\mathcal{C}|)}, r^{\left(\left|\mathcal{C}^{\prime}\right|\right)}\right]$.

Remark 2.7. This result shows that the condition that $m$ is even in Theorem 2.5 is important: the symmetric group $\mathbb{S}_{n}=G(1,1, n)$ does not have a mystical counterpart, and indeed the correspondence $\mu$ cannot be extended to groups $G(m, p, n)$ where $m$ is odd. This happens because the space of the invariants of $G(m, p, n)$ in $S(V)$ is not closed under the multiplication in $S_{-1}(V)$, and therefore cannot be the space of invariants of a group acting by automorphisms of $S_{-1}(V)$.

The groups $G=G(m, p, n)$ and $\mu(G)$ are of the same order $\frac{m^{n} n !}{p}$, and, informally, they "look very similar". Our next main result makes this informal statement more precise. We keep the notation from Theorem 2.5 and denote by $R$ the ring $\mathbb{Z}\left[\frac{1+\mathbf{i}}{2}\right] \subset \mathbb{C}$.

Main Theorem 2.8. For all $G$ as in Theorem 2.5, the group rings $R G$ and $R \mu(G)$ are isomorphic. In particular, $\mathbb{C} G \cong \mathbb{C} \mu(G)$.

This theorem is rather nontrivial because the groups $G$ and $\mu(G)$ are often not isomorphic as abstract groups. Indeed, it was shown in [2, Example 7.3] that the group $G=G(2,2, n)$ is not isomorphic to its mystic counterpart $\mu(G)=W_{\{ \pm 1\},\{1\}}$ for all even $n$. We generalize this observation and give a complete list of cases where $G$ is not isomorphic to $\mu(G)$.

Theorem 2.9. In the notation of Theorem 2.5 , let $G=G(m, p, n)$ with $m$ even. Then the groups $G$ and $\mu(G)$ are not isomorphic as abstract groups, if and only if $n$ is even and $\frac{m}{p}$ is odd.

We go further than this and classify all groups of the form $G=G(m, p, n)$ and $\mu(G)$ up to isomorphism. We need the following useful notion.

Definition 2.10. We say that a subgroup $G$ of a semidirect product $T \rtimes H$ is thick if

- $\pi(G)=H$, where $\pi: T \rtimes H \rightarrow H$ is the canonical projection onto the second factor;

- $G$ is normal in in $T \rtimes H$.

It is not difficult to see that all the groups from Theorem 2.5 are thick subgroups of $G(m, 1, n)=\mathcal{C}^{n} \rtimes \mathbb{S}_{n}$. It turns out that a converse is also true.

Theorem 2.11. Let $\mathcal{C}$ be the subgroup of $\mathbb{C}^{\times}$of order $m$. Then every thick subgroup of $\mathcal{C}^{n} \rtimes \mathbb{S}_{n}$ is of the form $G(m, p, n)$ or (if $m$ is even) $W_{\mathcal{C}, \mathcal{C}^{\prime}}$, and in particular, is a mystic reflection group.

The following completes the classification of thick subgroups of all $G(m, 1, n)$ up to isomorphism. We keep the notation used in the preceding theorems.

Theorem 2.12. Let $G \subseteq G(m, 1, n), G^{\prime} \subseteq G\left(m^{\prime}, 1, n^{\prime}\right)$ be thick subgroups.

(a) Suppose that $n=n^{\prime}$ and $G \neq G^{\prime}$ in $\mathbb{G}_{n}$. Then $G \cong G^{\prime}$ if and only if $n$ is odd, $m=m^{\prime}$ is even, and $\left\{G, G^{\prime}\right\}=\{G(m, p, n), \mu(G(m, p, n))\}$ for some $p$ such that $\frac{m}{p}$ is odd.

(b) Suppose that $n<n^{\prime}$. Then $G \cong G^{\prime}$ if and only if $n=3, n^{\prime}=4, G \in\{G(2,2,3), \mu(G(2,2,3))\}$, $G^{\prime}=G(1,1,4)=\mathbb{S}_{4}$.

Remark 2.13. It is not difficult to see that if $\frac{m}{p}$ is even (in the notation of Theorem 2.5), then $G=\mu(G)$ in $\mathbb{G}_{n}$. This theorem together with Theorem 2.8 implies that the converse is also true.

The above classification suggests the following general problem which we do not address in the present paper:

Problem 2.14. Given a semidirect product group $T \rtimes H$ where $T$ and $H$ are finite, classify all finite thick subgroups of $T \rtimes H$ up to isomorphism. 


\section{Proofs of results from Section 2}

We will repeatedly use the following straightforward technical fact about the root system of type $A_{n-1}$, the proof of which is left to the reader as an exercise.

Claim 3.1. Let $A$ be a multiplicatively written abelian group. Let $\phi_{i j}: \mathbb{Z}^{n} \rightarrow A, 1 \leq i, j \leq n$, $i \neq j$, be a system of maps satisfying

$$
\phi_{i j}(w(\mathbf{k}))=\phi_{w^{-1}(i), w^{-1}(j)}(\mathbf{k}), \quad \phi_{j i}(\mathbf{k}) \phi_{i j}(\mathbf{k})=1
$$

for all $\mathbf{k}=\left(k_{1}, \ldots, k_{n}\right) \in \mathbb{Z}^{n}, w \in \mathbb{S}_{n}$, where $w(\mathbf{k})$ stands for $\left(k_{w^{-1}(1)}, \ldots, k_{w^{-1}(n)}\right)$. Denote

$$
\phi_{w}(\mathbf{k})=\prod_{i<j, w(i)>w(j)} \phi_{i j}(\mathbf{k}) .
$$

Then:

(a) For all $w^{\prime}, w \in \mathbb{S}_{n}$,

$$
\phi_{w^{\prime} w}(\mathbf{k})=\phi_{w^{\prime}}(w(\mathbf{k})) \phi_{w}(\mathbf{k})
$$

(b) If for all $i \neq j$ and for all $\mathbf{k}, \mathbf{k}^{\prime} \in \mathbb{Z}^{n}$ one has

$$
\phi_{i j}\left(\mathbf{k}+\mathbf{k}^{\prime}\right)=a_{i j}^{k_{i} k_{j}^{\prime}-k_{i}^{\prime} k_{j}} \phi_{i j}(\mathbf{k}) \phi_{i j}\left(\mathbf{k}^{\prime}\right)
$$

then for all $w \in \mathbb{S}_{n}$ one has

$$
\left\langle\mathbf{k}, \mathbf{k}^{\prime}\right\rangle \phi_{w}\left(\mathbf{k}+\mathbf{k}^{\prime}\right)=\left\langle w(\mathbf{k}), w\left(\mathbf{k}^{\prime}\right)\right\rangle \phi_{w}(\mathbf{k}) \phi_{w}\left(\mathbf{k}^{\prime}\right),
$$

where $\left\langle\mathbf{k}, \mathbf{k}^{\prime}\right\rangle=\prod_{i<j} a_{i j}^{k_{i}^{\prime} k_{j}}$. Here $a_{i j}$ are elements of $A$ such that $a_{j i}=a_{i j}$.

\section{Proof of Proposition 2.3}

Observe that the group $\mathbb{G}_{n} \subset \mathrm{GL}_{n}(\mathbb{C})$ is generated by $\mathbb{S}_{n}$ and $\left(\mathbb{C}^{\times}\right)^{n}$ subject to the semidirect product relations

$$
w t_{j}^{(\zeta)}=t_{w(j)}^{(\zeta)} w, \quad \text { for all } w \in \mathbb{S}_{n}, \quad j \in\{1, \ldots, n\}, \quad \zeta \in \mathbb{C}^{\times} .
$$

In what follows, we use the abbreviation $x^{\mathbf{k}}$ to denote $x_{1}^{k_{1}} \cdots x_{n}^{k_{n}}$, where $\mathbf{k}=\left(k_{1}, \ldots, k_{n}\right) \in \mathbb{Z}_{\geq 0}^{n}$. For $c \in \mathbb{C}^{\times}$, define $\phi_{i j}^{(c)}: \mathbb{Z}^{n} \rightarrow \mathbb{C}^{\times}$by

$$
\phi_{i j}^{(c)}(\mathbf{k})=(-1)^{k_{i} k_{j}} c^{\bar{k}_{i}-\bar{k}_{j}}, \quad \text { where } \quad \bar{k}=(k \bmod 2) \in\{0,1\} .
$$

Additionally, define $\phi_{i j}^{(0)}(\mathbf{k})=1$ for all $\mathbf{k}$. Because $\phi_{i j}^{\left(c^{-1}\right)}(\mathbf{k})=\phi_{i j}^{(c)}(\mathbf{k})^{-1}=\phi_{j i}^{(c)}(\mathbf{k})$, the system $\phi_{i j}^{(c)}$ satisfies the condition in Claim 3.1 and hence gives rise to functions $\phi_{w}^{(c)}$. The following lemma is then immediate from Claim 3.1(a).

Lemma 3.2. For each $c \in \mathbb{C}$, the formula

$$
w t_{1}^{\left(\zeta_{1}\right)} \cdots t_{n}^{\left(\zeta_{n}\right)} \triangleright_{c} x^{\mathbf{k}}=\phi_{w}^{(c)}(\mathbf{k})\left(\prod_{j=1}^{n} \zeta_{j}^{k_{j}}\right) x^{w(\mathbf{k})}
$$

defines an action $\triangleright_{c}$ of the group $\mathbb{G}_{n}$ on the space $S(V)$. 
The actions $\triangleright_{0}, \triangleright_{1}$ given by Lemma 3.2 coincide on the generators $s_{i}, t_{j}^{(\zeta)}$ of $\mathbb{G}_{n}$ with the actions $\triangleright_{+}, \triangleright_{-}$defined in part (a) of Proposition 2.3. Hence part (a) of the proposition is proved.

Denote by $\bullet_{+}$, respectively $\bullet_{-}$, the multiplication on the algebra $S(V)$, respectively $S_{-\mathbf{1}}(V)$. One has the following multiplication rule for monomials:

$$
x^{\mathbf{k}} \bullet x^{\mathbf{k}^{\prime}}=\left\langle\mathbf{k}, \mathbf{k}^{\prime}\right\rangle_{ \pm} x^{\mathbf{k}+\mathbf{k}^{\prime}} \quad \text { for all } \mathbf{k}, \mathbf{k}^{\prime} \in \mathbb{Z}_{\geq 0}^{n},
$$

where $\left\langle\mathbf{k}, \mathbf{k}^{\prime}\right\rangle_{ \pm}$is as given in Claim 3.1(b) with all $a_{i j}= \pm 1, i \neq j$.

It is enough to check that $w \triangleright_{ \pm}$and $t_{j}^{(\zeta)} \triangleright_{ \pm}$are automorphisms of the respective algebra structures on $S(V)$. We apply these actions to both sides of the multiplication rule for monomials and check that the results are equal. This is trivial for $t_{j}^{(\zeta)} \triangleright_{ \pm}$. For $w \triangleright_{ \pm}$where $w \in \mathbb{S}_{n}$, the equality is guaranteed by Claim 3.1(b) and Lemma 3.2, applied to functions $\phi_{i j}^{+}=\phi_{i j}^{(0)}$, respectively $\phi_{i j}^{-}=\phi_{i j}^{(1)}$. This proves parts (b), (c) of Proposition 2.3.

Now let us prove part $(\mathrm{d})$ of Proposition 2.3. Clearly, the natural $\mathbb{S}_{n}$-action on the group $\left(\mathbb{C}^{\times}\right)^{n} \subset \mathbb{G}_{n}$ extends to that on the group algebra $\mathbb{C}\left(\mathbb{C}^{\times}\right)^{n}$.

For each $c \in \mathbb{C} \backslash\{0\}, w \in \mathbb{S}_{n}$, define the element $Q_{w}^{(c)} \in \mathbb{C}\left(\mathbb{C}^{\times}\right)^{n}$ by

$$
Q_{w}^{(c)}=\prod_{i<j: w(i)>w(j)} Q_{i j}^{(c)}
$$

where $Q_{i j}^{(c)}=\frac{1}{4}\left(\left(c+c^{-1}\right)\left(1-t_{i}^{(-1)} t_{j}^{(-1)}\right)+\left(c-c^{-1}+2\right) t_{i}^{(-1)}+\left(c^{-1}-c+2\right) t_{j}^{(-1)}\right), 1 \leq i, j \leq n$.

Lemma 3.3. For all $w^{\prime}, w \in \mathbb{S}_{n}, c \in \mathbb{C}^{\times}, Q_{w}^{(c)} Q_{w}^{\left(c^{-1}\right)}=1$ and $Q_{w^{\prime} w}^{(c)}=w^{-1}\left(Q_{w^{\prime}}^{(c)}\right) \cdot Q_{w}^{(c)}$.

Proof. Denote by $\mathcal{F}$ the algebra of all functions from $\mathbb{Z}^{n}$ to $\mathbb{C}$ with pointwise addition and multiplication. Clearly, the assignment $t_{1}^{\left(\zeta_{1}\right)} \ldots t_{n}^{\left(\zeta_{n}\right)} \mapsto\left(\mathbf{k} \mapsto \zeta_{1}^{k_{1}} \ldots \zeta_{n}^{k_{n}}\right)$ defines a group homomorphism $\left(\mathbb{C}^{\times}\right)^{n} \rightarrow \mathcal{F}^{\times}$which extends to an injective map $\Psi: \mathbb{C}\left(\mathbb{C}^{\times}\right)^{n} \hookrightarrow \mathcal{F}$.

It is easy to check that $\Psi\left(Q_{i j}^{(c)}\right)=\phi_{i j}^{(c)}$ where $\phi_{i j}^{(c)}$ is as in Lemma 3.2. In particular, $Q_{i j}^{(c)} Q_{i j}^{\left(c^{-1}\right)}=1$ since $\phi_{i j}^{(c)} \phi_{i j}^{\left(c^{-1}\right)}=1$ and $\Psi$ is injective. This proves the first assertion of the lemma. To prove the second assertion, apply $\Psi^{-1}$ to Claim 3.1(a) and use the fact that the function $\mathbf{k} \mapsto \phi(w(\mathbf{k}))$ is mapped by $\Psi^{-1}$ to $w^{-1}\left(\Psi^{-1}(\phi)\right)$ for all functions $\phi$ from the subgroup of $\mathcal{F}^{\times}$generated by $\left\{\phi_{i j}^{(c)}: i \neq j\right\}$.

Now for each $c \in \mathbb{C}^{\times}$define the $\mathbb{C}$-linear map $J_{c}: \mathbb{C}_{n} \rightarrow \mathbb{C} \mathbb{G}_{n}$ by the formula

$$
J_{c}(w t)=w t Q_{w}^{(c)}, \quad t \in \mathbb{C}\left(\mathbb{C}^{\times}\right)^{n}, \quad w \in \mathbb{S}_{n} .
$$

Lemma 3.4. For each $n \geq 1$,

(a) $J_{c}$ is an algebra automorphism of $\mathbb{C}_{n}$ with inverse $J_{c^{-1}}$.

(b) $\rho_{+} \circ J_{c}=\rho_{c}$, where $\rho_{c}: \mathbb{C}_{n} \rightarrow \operatorname{End}_{\mathbb{C}} S(V)$ is the algebra homomorphism corresponding to $\triangleright_{c}$.

Proof. On the one hand, $J_{c}\left(w^{\prime} t^{\prime} w t\right)=J_{c}\left(w^{\prime} w \cdot w^{-1}\left(t^{\prime}\right) t\right)=w^{\prime} w \cdot w^{-1}\left(t^{\prime}\right) t Q_{w^{\prime} w}^{(c)}$. On the other hand,

$$
J_{c}\left(w^{\prime} t^{\prime}\right) J_{c}(w t)=w^{\prime} t^{\prime} Q_{w^{\prime}}^{(c)} w t Q_{w}^{(c)}=w^{\prime} w \cdot w^{-1}\left(t^{\prime}\right) t w^{-1}\left(Q_{w^{\prime}}^{(c)}\right) Q_{w}^{(c)}=J_{c}\left(w^{\prime} t^{\prime} w t\right)
$$

by the second assertion of Lemma 3.3. Hence $J_{c}$ is a homomorphism of algebras. Now

$$
J_{c}\left(J_{c^{-1}}(w t)\right)=J_{c}\left(w t Q_{w}^{\left(c^{-1}\right)}\right)=w t Q_{w}^{\left(c^{-1}\right)} Q_{w}^{(c)}=w t
$$

by the first assertion of Lemma 3.3. This proves part (a) of the lemma. 
Prove (b). In view of Lemma 3.2, it suffices to show that $Q_{w}^{(c)} \triangleright_{+} x^{\mathbf{k}}=\phi_{w}^{(c)}(\mathbf{k}) x^{\mathbf{k}}$. Indeed,

$$
\begin{aligned}
Q_{w}^{(c)} \triangleright_{+} x^{\mathbf{k}} & =\frac{1}{4}\left(\left(c+c^{-1}\right)\left(1-(-1)^{k_{i}+k_{j}}\right)+\left(c-c^{-1}+2\right)(-1)^{k_{i}}+\left(c^{-1}-c+2\right)(-1)^{k_{j}}\right) x^{\mathbf{k}} \\
& =\phi_{i j}^{(c)}(\mathbf{k}) x^{\mathbf{k}} .
\end{aligned}
$$

Finally,

$$
J_{c}(w t) \triangleright_{+} x^{\mathbf{k}}=\left(w t Q_{w}^{(c)}\right) \triangleright_{+} x^{\mathbf{k}}=(w t) \triangleright_{+}\left(Q_{w}^{(c)} \triangleright_{+} x^{\mathbf{k}}\right)=\phi_{w}^{(c)}(\mathbf{k})(w t) \triangleright_{+} x^{\mathbf{k}}=w t \triangleright_{c} x^{\mathbf{k}} .
$$

Taking $c=1$ in Lemma 3.4(b), we settle Proposition 2.3(d). Proposition 2.3 is proved.

\section{Proof of Theorem 2.5}

We retain the notation from the proof of Proposition 2.3. Let $G=G(m, p, n)$ as in the theorem, and denote $T:=G \cap\left(\mathbb{C}^{\times}\right)^{n}$. Let $e_{T}=\sum_{t \in T} t \in \mathbb{C} T \subset \mathbb{C} G$. Clearly, $t_{i}^{(-1)} e_{T}=t_{1}^{(-1)} e_{T}$ for all $i=1, \ldots, n$. Hence $Q_{i j}^{(c)} e_{T}=t_{1}^{(-1)} e_{T}$ for all $c \in \mathbb{C}^{\times}$, so that $Q_{w}^{(c)} e_{T}=t_{1}^{(\operatorname{det} w)} e_{T}$ for all $w \in \mathbb{S}_{n}$. Since, as sets, $G=\left\{w t \mid w \in \mathbb{S}_{n}, t \in T\right\}$, one has $e_{G}=\sum_{w \in \mathbb{S}_{n}} w \cdot e_{T}$ and

$$
J_{c}\left(e_{G}\right)=\sum_{w \in \mathbb{S}_{n}} J_{c}(w) e_{T}=\sum_{w \in \mathbb{S}_{n}} w Q_{w}^{(c)} e_{T}=\sum_{w \in \mathbb{S}_{n}} w t_{1}^{(\operatorname{det} w)} e_{T}=e_{\mu(G)}
$$

because, as sets, $\mu(G)=\left\{w t_{1}^{(\operatorname{det} w)} t \mid w \in \mathbb{S}_{n}, t \in T\right\}$. Since a subgroup $G^{\prime}$ of $\mathbb{G}_{n}$ is uniquely determined by $e_{G^{\prime}} \in \mathbb{C}_{n}$, the group $\mu(G)$ is a unique subgroup $G^{\prime}$ of $\mathbb{G}_{n}$ such that $J_{c}\left(e_{G}\right)=e_{G^{\prime}}$.

Finally, by Lemma 3.4(b), $\rho_{c}\left(e_{G}\right)=\rho_{+}\left(e_{\mu(G)}\right)$. Setting $c=1$ and using injectivity of $\rho_{+}$, see Remark 2.4, completes the proof of Theorem 2.5.

\section{Proof of Theorem 2.8}

Let $G=G(m, p, n)$. Consider the restriction of $J_{\mathbf{i}}, \mathbf{i}=\sqrt{-1}$ to $R G=\mathbb{S}_{n} \cdot R T$ where $T=$ $G \cap\left(\mathbb{C}^{\times}\right)^{n}=\mu(G) \cap\left(\mathbb{C}^{\times}\right)^{n}$. Observe that $J_{\mathbf{i}}(T)=T$ and

$$
J_{\mathbf{i}}\left(s_{i}\right)=s_{i} Q_{s_{i}}^{(\mathbf{i})}=s_{i} Q_{i, i+1}^{(\mathbf{i})}=\frac{1}{2} s_{i}\left((1+\mathbf{i}) t_{i}^{(-1)}+(1-\mathbf{i}) t_{i+1}^{(-1)}\right)=\frac{1+\mathbf{i}}{2} \sigma_{i}+\frac{1-\mathbf{i}}{2} \sigma_{i}^{-1},
$$

where $\sigma_{i}=s_{i} t_{i}^{(-1)} \in \mu(G)$. Thus, $J_{\mathbf{i}}(R G) \subseteq R \mu(G)$, so that the automorphism $J_{\mathbf{i}}$ of $\mathbb{C G}_{n}$ restricts to an isomorphism $R G \stackrel{\sim}{\rightarrow} R \mu(G)$. Theorem 2.8 is proved.

\section{Proof of Theorem 2.11}

It is convenient to prove Theorem 2.11 before Theorems 2.9 and 2.12. We start with the following lemma.

Lemma 3.5. Let $G$ be a thick subgroup of $G(m, 1, n)=\mathcal{C}^{n} \rtimes \mathbb{S}_{n}$ where $\mathcal{C}$ is the subgroup of $\mathbb{C}^{\times}$ of order $m$. Then the group $T=G \cap \mathcal{C}^{n}$ is of the form $T_{\mathcal{C}, \mathcal{C}^{\prime}}=\left\{t \in \mathcal{C}^{n}: \operatorname{det} t \in \mathcal{C}^{\prime}\right\}$ for some subgroup $\mathcal{C}^{\prime} \subset \mathcal{C}$ of $\mathbb{C}^{\times}$, and is generated by $\left\{t_{1}^{\left(\epsilon^{\prime}\right)}: \epsilon^{\prime} \in \mathcal{C}^{\prime}\right\} \cup\left\{t_{i}^{(\epsilon)} t_{j}^{\left(\epsilon^{-1}\right)}: \epsilon \in \mathcal{C}, 1 \leq i, j \leq n\right\}$.

Proof. Let $\epsilon$ be a generator of $\mathcal{C}$, so that $t_{1}^{(\epsilon)} \in G(m, 1, n)$. Since $G$ is thick, there is an element in $G$ of the form $t s_{1}$ where $t \in \mathcal{C}^{n}$. By the normality of $G, t_{1}^{(\epsilon)}\left(t s_{1}\right) t_{1}^{\left(\epsilon^{-1}\right)}\left(t s_{1}\right)^{-1}=t_{1}^{(\epsilon)} t_{2}^{\left(\epsilon^{-1}\right)}$ belongs to $G$, hence to $T$. Because $\mathbb{S}_{n}$ acts on $T$ (by conjugation within $G$ ), it follows that $t_{i}^{(\epsilon)} t_{j}^{\left(\epsilon^{-1}\right)} \in T$. These elements generate the subgroup $T_{0}=T_{\mathcal{C},\{1\}}$ of $T$. 
Every element $t^{\prime} \in T$ is equal, modulo $T_{0}$, to an element of the form $t_{1}^{\left(\epsilon^{\prime}\right)}$ for some $\epsilon^{\prime} \in \mathcal{C}$, where $\epsilon^{\prime}=\operatorname{det} t^{\prime}$. Denote by $\mathcal{C}^{\prime}$ the group formed by all such $\epsilon^{\prime}$. Then $T \subseteq T_{\mathcal{C}, \mathcal{C}^{\prime}}$, and, since $\left\{t_{1}^{\left(\epsilon^{\prime}\right)}: \epsilon^{\prime} \in \mathcal{C}^{\prime}\right\} \cup T_{0}$ generates $T_{\mathcal{C}, \mathcal{C}^{\prime}}$, one has $T \supseteq T_{\mathcal{C}, \mathcal{C}^{\prime}}$.

We continue the proof of Theorem 2.11. Let $G$ be a thick subgroup of $G(m, 1, n)=\mathcal{C}^{n} \rtimes \mathbb{S}_{n}$ so that $G \cap \mathcal{C}^{n}=T_{\mathcal{C}, \mathcal{C}^{\prime}}$ as in Lemma 3.5. Because $G$ is thick, $G$ contains an element of the form $t s_{1}$ where $t \in \mathcal{C}^{n}$. Premultiplying $t s_{1}$ by an element of $T_{\mathcal{C},\{1\}}$, we conclude that $G \ni t_{1}^{(\epsilon)} s_{1}$, hence $G$ contains $\left(t_{1}^{(\epsilon)} s_{1}\right)^{2}=t_{1}^{(\epsilon)} t_{2}^{(\epsilon)}$. It follows that $t_{1}^{(\epsilon)} t_{2}^{(\epsilon)} \in T_{\mathcal{C}, \mathcal{C}^{\prime}}$, hence $\operatorname{det} t_{1}^{(\epsilon)} t_{2}^{(\epsilon)}=\epsilon^{2} \in \mathcal{C}^{\prime}$. This means that

- either $\epsilon \in \mathcal{C}^{\prime}$, implying $t_{1}^{(\epsilon)} \in G$ and $s_{1} \in G$;

- or $-\epsilon \in \mathcal{C}^{\prime}$, implying $t_{1}^{(-\epsilon)} \in G$ and $t_{1}^{(-1)} s_{1} \in G$.

We note the following easy lemma.

Lemma 3.6. Let $G$ be a normal subgroup of $\mathcal{C}^{n} \rtimes \mathbb{S}_{n}=G(m, 1, n)$ such that $G \cap \mathcal{C}^{n}=T_{\mathcal{C}, \mathcal{C}^{\prime}}$.

(a) If $G \ni s_{1}$, then $G$ contains all elements of the form $t w$ with $t \in T_{\mathcal{C}, \mathcal{C}^{\prime}}$ and $w \in \mathbb{S}_{n}$.

(b) If $G \ni t_{1}^{(-1)} s_{1}$, then $G$ contains all elements $t t_{1}^{(\operatorname{det} w)} w$ with $t \in T_{\mathcal{C}, \mathcal{C}^{\prime}}$ and $w \in \mathbb{S}_{n}$.

To continue the proof of Theorem 2.11, suppose $s_{1} \in G$. Then by Lemma 3.6, $G \supseteq \mathbb{S}_{n}$, Hence $G=\left(G \cap \mathcal{C}^{n}\right) \rtimes \mathbb{S}_{n}=T_{\mathcal{C}, \mathcal{C}^{\prime}} \rtimes \mathbb{S}_{n}$. Then $G=G(m, p, n)$ is a complex reflection group with $\frac{m}{p}=\left|\mathcal{C}^{\prime}\right|$.

The only remaining case is $s_{1} \notin G$ but $t_{1}^{(-1)} s_{1} \in G$. It follows from Lemma 3.6 that $t^{\prime} w \in G$ (where $t^{\prime} \in \mathcal{C}^{n}$ and $w \in \mathbb{S}_{n}$ ), if and only if $t^{\prime} t_{1}^{(\operatorname{det} w)} \in G$, if and only if $t^{\prime} t_{1}^{(\operatorname{det} w)} \in G \cap \mathcal{C}^{n}=T_{\mathcal{C}, \mathcal{C}^{\prime}}$, if and only if $\operatorname{det}\left(t^{\prime} t_{1}^{(\operatorname{det} w)}\right) \in \mathcal{C}^{\prime}$. Observing that $\operatorname{det}\left(t^{\prime} t_{1}^{(\operatorname{det} w)}\right)=\operatorname{det}\left(t^{\prime} w\right)$, we obtain

$$
G=\left\{t^{\prime} w \in \mathcal{C}^{n} \rtimes \mathbb{S}_{n}: \operatorname{det}\left(t^{\prime} w\right) \in \mathcal{C}^{\prime}\right\}=W_{\mathcal{C}, \mathcal{C}^{\prime}}
$$

In this case, $t_{1}^{(-1)} s_{1} \in \mathcal{C}^{n} \rtimes \mathbb{S}_{n}$ means that $-1 \in \mathcal{C}$. That is, $m$ is even. Theorem 2.11 is proved.

\section{Proof of Theorem 2.9}

We will use the following notion.

Definition 3.7. A thick subgroup $G$ of $G(m, 1, n)$ is regular, if for each normal abelian subgroup $N$ of $G$, either $N=T_{G}:=G \cap\left(\mathbb{C}^{\times}\right)^{n}$ or $|N|<\left|T_{G}\right|$. Otherwise, $G$ is singular.

\section{Lemma 3.8.}

(a) Suppose that $G_{i}$ is a regular thick subgroup of $G\left(m_{i}, 1, n_{i}\right), i=1,2$. If $G_{1}$ and $G_{2}$ are isomorphic (as abstract groups), then $m_{1}=m_{2}, n_{1}=n_{2}$ and $T_{G_{1}}=T_{G_{2}}$.

(b) A thick subgroup $G$ of $G(m, 1, n)$ is singular, if and only if $G$ belongs to the following list: $G(1,1, n)$ with $n=2,3,4, G(2,1,2), G(2,2,2), \mu(G(2,2,2))$.

Proof. (a) Since $G_{i}$ is regular and $T_{G_{i}}$ is the unique largest order normal abelian subgroup of $G_{i}$, the restriction of any isomorphism $f: G_{1} \rightarrow G_{2}$ to $T_{G_{1}}$ is an isomorphism $T_{G_{1}} \stackrel{\sim}{\rightarrow} T_{G_{2}}$, and $f$ induces an isomorphism $\bar{f}: \mathbb{S}_{n_{1}}=G_{1} / T_{G_{1}} \rightarrow \mathbb{S}_{n_{2}}=G_{2} / T_{G_{2}}$. Hence $n_{1}=n_{2}$. Furthermore, $m_{i}$ is the exponent of the group $T_{G_{i}}$, hence $m_{1}=m_{2}$. Finally, $T_{G_{i}}=T_{\mathcal{C}, \mathcal{C}_{i}^{\prime}}$ by Lemma 3.5, and $\left|T_{G_{i}}\right|=m_{i}^{n_{i}-1}\left|\mathcal{C}_{i}^{\prime}\right|$ implies $\mathcal{C}_{1}^{\prime}=\mathcal{C}_{2}^{\prime}$. This proves part (a).

(b) Clearly, the subgroup $T:=T_{G}=T_{\mathcal{C}, \mathcal{C}^{\prime}}$ as in Lemma 3.5 is a normal abelian subgroup of $G$. Let $N$ be a normal abelian subgroup of $G$. We will show that $N \subseteq T$. 
The map $\pi: G \rightarrow \mathbb{S}_{n}$ is surjective as $G$ is thick, therefore $\pi(N)$ is a normal abelian subgroup of $\mathbb{S}_{n}$. Hence if $n=1$ or $n \geq 5, \pi(N)$ can only be $\{1\}$ so $N \subseteq \operatorname{ker} \pi=T$ and $G$ is regular.

Let $2 \leq n \leq 4$. Then $\mathbb{S}_{n}$ has a unique normal abelian subgroup $K$ which is not $\{1\}$. Assume that $N \not \subset T$. Then $\pi(N)=K$. One can check that $K$ acts on the indices $1, \ldots, n$ transitively, hence the centralizer of $K$ in $T$ is the set of scalar matrices in $T$, which is the center $C(G)$ of $G$. Because the conjugation action of $N$ on $T$ factors through $\pi(N)$, we have $C_{G}(N) \cap T=C(G)$. But $N$ is abelian, so $N \subseteq C_{G}(N)$. Thus, $N \cap T \subset C(G)$.

Now let $w t \in N$ where $1 \neq w \in \mathbb{S}_{n}$ and $t \in\left(\mathbb{C}^{\times}\right)^{n}$. Let $i \in\{1, \ldots, n\}$ be such that $w(i) \neq i$, and let $\epsilon$ be a generator of $\mathcal{C}$. Then $N \ni t_{i}^{(\epsilon)} \cdot w t \cdot t_{i}^{\left(\epsilon^{-1}\right)} \cdot(w t)^{-1}=t_{i}^{(\epsilon)} t_{w(i)}^{\left(\epsilon^{-1}\right)}$ which is a scalar matrix only if $m=1$ or $m=n=2$.

To prove the if part of Theorem 2.9 , let $m, n$ be even, $\frac{m}{p}$ be odd, and $\mathcal{C}^{\prime}, \mathcal{C}$ be subgroups of $\mathbb{C}^{\times}$of order $\frac{m}{p}, m$, respectively. Assume for contradiction that there is an isomorphism $\varphi: W_{\mathcal{C}, \mathcal{C}^{\prime}} \rightarrow G(m, p, n)$.

In the case $G=G(2,2,2)=T_{\{ \pm 1\},\{1\}} \times \mathbb{S}_{2}, G$ is a Klein 4-group which is not isomorphic to $W_{\{ \pm 1\},\{1\}}$, a cyclic group generated by $s_{1} t_{1}^{(-1)}$ of order 4 .

In all other cases, by Lemma $3.5 T=T_{\mathcal{C}, \mathcal{C}^{\prime}}$ is the unique maximal normal abelian subgroup of $W_{\mathcal{C}, \mathcal{C}^{\prime}}$ and of $G=G(m, p, n)$, hence $\varphi(T)=T$ and $\varphi$ induces an isomorphism $\bar{\varphi}: \mathbb{S}_{n}=$ $\mu(G) / T \rightarrow \mathbb{S}_{n}=G / T$. Let us state three easy lemmas, in which we refer to a cycle of length $n$ in $\mathbb{S}_{n}$ as a long cycle.

Lemma 3.9. Let $n$ be even and $c \in \mathbb{S}_{n}$ be a long cycle. Then $\operatorname{det}\left(t_{1}^{(-1)} c\right)=1$ and $t_{1}^{(-1)} c \in W_{\mathcal{C}, \mathcal{C}^{\prime}}$. Lemma 3.10. For $t \in\left(\mathbb{C}^{\times}\right)^{n}$ and a long cycle $c,(t c)^{n}=z^{(\operatorname{det} t)}$, where $z^{(\epsilon)}$ denotes $t_{1}^{(\epsilon)} t_{2}^{(\epsilon)} \cdots t_{n}^{(\epsilon)}$.

Lemma 3.11. The image of a long cycle in $\mathbb{S}_{n}$ under any automorphism of $\mathbb{S}_{n}$ is a long cycle.

Let $c$ be a long cycle in $\mathbb{S}_{n}$. By Lemma $3.9, \bar{c}=t_{1}^{(-1)} c \in \mu(G)$. By Lemma 3.10, $(\bar{c})^{n}=z^{(-1)}$. Hence $(\bar{c})^{n\left|\mathcal{C}^{\prime}\right|}=\left(z^{(-1)}\right)^{\left|\mathcal{C}^{\prime}\right|}=z^{(-1)}$ as $\left|\mathcal{C}^{\prime}\right|=\frac{m}{p}$ is odd.

Now consider $\varphi(\bar{c}) \in G$. It is of the form $t \bar{\varphi}(c)$, where $t$ is some element of $\mathcal{C}^{n}$ such that, by definition of $G=G(m, p, n)$, $\operatorname{det} t \in \mathcal{C}^{\prime}$. By Lemma 3.11, $\bar{\varphi}(c)$ is a long cycle in $\mathbb{S}_{n}$. Therefore, by Lemma 3.10 ,

$$
(\varphi(\bar{c}))^{n\left|\mathcal{C}^{\prime}\right|}=\left(z^{(\operatorname{det} t)}\right)^{\left|\mathcal{C}^{\prime}\right|}=z^{\left((\operatorname{det} t)^{\left|\mathcal{C}^{\prime}\right|}\right)}=1 .
$$

This is a contradiction, because the image of $z^{(-1)} \neq 1$ under an isomorphism $\varphi$ cannot be 1 . The if part of Theorem 2.9 is proved.

To establish the only if part of Theorem 2.9, observe that if $\frac{m}{p}$ is even, then the groups $G=G(m, p, n)$ and $\mu(G)=W_{\mathcal{C}, \mathcal{C}^{\prime}}$ simply coincide as subgroups of $\mathbb{G}_{n}$. Indeed, in this case $\operatorname{det} w \in\{ \pm 1\} \subseteq \mathcal{C}^{\prime}$, hence the conditions $\operatorname{det} t \in \mathcal{C}^{\prime}$ and $\operatorname{det} t w \in \mathcal{C}^{\prime}$ are equivalent.

If $m$ is even, $\frac{m}{p}$ is odd and $n$ is odd, both $G=G(m, p, n)$ and $\mu(G)$ are normal subgroups of $\widetilde{G}=G\left(m, \frac{p}{2}, n\right)$ which do not contain the central subgroup $Z=\left\{1, z^{(-1)}\right\}$ of $\widetilde{G}$. Hence $\widetilde{G}=G \times Z=\mu(G) \times Z$, so that $G \cong \mu(G) \cong \widetilde{G} / Z$. Theorem 2.9 is proved.

\section{Proof of Theorem 2.12}

Let $G \subseteq G(m, 1, n), G^{\prime} \subseteq G\left(m^{\prime}, 1, n^{\prime}\right)$ be thick subgroups.

Assume that $G \neq G^{\prime}$ are regular in the sense of Definition 3.7. Then by Lemma 3.8(a), $G \cong G^{\prime}$ implies $n=n^{\prime}, m=m^{\prime}$ and $T_{G}=T_{G^{\prime}}$, so we are done by Theorem 2.11.

By inspection of the list in Lemma 3.8(b), no two singular subgroups are isomorphic to each other. Also by inspection, one shows that if $n=n^{\prime}, G$ is singular and $G^{\prime}$ is regular, then $G$ and $G^{\prime}$ are never isomorphic. 
The only remaining case is when $n \neq n^{\prime}, G$ is singular and $G^{\prime}$ is regular. If $G \cong G^{\prime}$, then $n, n^{\prime} \leq 4$, because $\left|\mathbb{S}_{5}\right|>|G|$. Then, an easy analysis based on the cardinalities of singular groups shows that the only possible isomorphism is the one given in part (b). Theorem 2.12 is proved.

\section{Appendix}

The aim of this section is to prove the following important result about group ring actions.

Theorem 4.1. Let $A$ be an integral domain and $G$ be a group of ring automorphisms of $A$. Then:

(a) the natural map $\rho: A G \rightarrow \operatorname{End}_{\mathbb{Z}}(A)$ given by $\left(\rho\left(\sum_{i} a_{i} g_{i}\right)\right)(a)=\sum_{i} a_{i} g_{i}(a)$ is injective;

(b) with respect to the natural ring structure on $\operatorname{End}_{\mathbb{Z}}(A)$ and the semidirect product structure $A \rtimes \mathbb{Z} G$ on $A G$, the map $\rho$ is a ring homomorphism.

The following is immediate from the theorem.

Corollary 4.2. In the notation of the theorem, let $R$ be a subring of $A^{G}$. Then the restriction of $\rho$ to $R G$ is an injective ring homomorphism

$$
R G \hookrightarrow \operatorname{End}_{R} A,
$$

where $R G$ is the ordinary group ring of $G$.

Proof of Theorem 4.1. We need the following generalization of the celebrated Dedekind's lemma.

Lemma 4.3. Let $A$ be an integral domain and $B$ be a multiplicative monoid. Let $\mathcal{G}$ be a set of monoid homomorphisms $B \rightarrow A$. Then the natural A-linear map

$$
\rho: A \mathcal{G} \rightarrow \operatorname{Fun}(B, A), \quad\left(\rho\left(\sum_{g} a_{g} g\right)\right)(b)=\sum_{g} a_{g} g(b)
$$

is injective. (Here $A \mathcal{G}=\oplus_{g \in \mathcal{G}} A g$ is the free $A$-module generated by $\mathcal{G}$.)

Proof. Assume for contradiction that $\rho$ is not injective. Let $\mathcal{G}_{0}$ be a minimal finite subset of $\mathcal{G}$ such that there exists a non-zero element $\sum_{g \in \mathcal{G}_{0}} a_{g} g$ in the kernel of $\rho$. That is,

$$
\sum_{g \in \mathcal{G}_{0}} a_{g} g(b)=0 \quad \text { for all } b \in B
$$

Clearly, all $a_{g}$ are non-zero and $\left|\mathcal{G}_{0}\right|>1$ because $A$ has no zero divisors. In particular, for each $b, b^{\prime} \in B$,

$$
\sum_{g \in \mathcal{G}_{0}} a_{g} g\left(b^{\prime} b\right)=\sum_{g \in \mathcal{G}_{0}} a_{g} g\left(b^{\prime}\right) g(b)=0,
$$

because all $g \in \mathcal{G}_{0}$ are homomorphisms from $B$ to $A$. Furthermore, fix $h \in \mathcal{G}_{0}$. Combining the above identities, we obtain

$$
\sum_{g \in \mathcal{G}_{0} \backslash\{h\}} a_{g} g\left(b^{\prime}\right)(g(b)-h(b))=0 \quad \text { for all } \quad b, b^{\prime} \in B .
$$


That is, for each $b \in B$, the element

$$
k_{b}:=\sum_{g \in \mathcal{G}_{0} \backslash\{h\}} a_{g}(g(b)-h(b)) g
$$

belongs to ker $\rho$. The minimality of $\mathcal{G}_{0}$ implies that $k_{b}=0$ for all $b \in B$, which is equivalent to $g(b)=h(b)$ for all $b \in B$ and all $g \in \mathcal{G}_{0}$. That is, $\left|\mathcal{G}_{0}\right|=1$, a contradiction.

We now use Lemma 4.3 for $A$ as in Theorem 4.1 with $B=A \backslash\{0\}$ and $\mathcal{G}=G$ viewed as homomorphisms of monoids $A \backslash\{0\} \rightarrow A$. Since $\operatorname{End}_{\mathbb{Z}} A$ is naturally a subset of $\operatorname{Fun}(A \backslash\{0\}, A)$ and $\rho(A G) \in \operatorname{End}_{\mathbb{Z}} A \subset \operatorname{Fun}(A \backslash\{0\}, A)$, part (a) of Theorem 4.1 is proved.

To prove (b), note that $\operatorname{End}_{\mathbb{Z}} A$ is naturally a ring, with multiplication being composition of maps. The semidirect product multiplication on $A G$ is given by the formula $(a g)\left(a^{\prime} g^{\prime}\right)=$ $a g\left(a^{\prime}\right) \cdot g g^{\prime}$ for all $a, a^{\prime} \in A, g, g^{\prime} \in G$. Then

$$
\rho\left((a g)\left(a^{\prime} g^{\prime}\right)\right)(b)=a \cdot g\left(a^{\prime} \cdot g^{\prime}(b)\right)=a \cdot g\left(a^{\prime}\right) \cdot g\left(g^{\prime}(b)\right)=a g\left(a^{\prime}\right)\left(g g^{\prime}\right)(b)
$$

for all $a, a^{\prime}, b \in A, g, g^{\prime} \in G$. Part (b) of the theorem is proved.

\section{Acknowledgments}

We thank Ken Brown for bringing the paper [2] to our attention, and Alexander Premet for stimulating discussions. The present paper was started when both authors were research members of the Mathematical Sciences Research Institute. We thank the Institute and the organizers of the Noncommutative Algebraic Geometry and Representation Theory program for creating an atmosphere conducive for research. We acknowledge partial support of the LMS Research in Pairs grant ref. 41224. The second named author was partially supported by the NSF grant DMS-1101507.

\section{References}

[1] Bazlov Y., Berenstein A., Noncommutative Dunkl operators and braided Cherednik algebras, Selecta Math. (N.S.) 14 (2009), 325-372, arXiv:0806.0867.

[2] Kirkman E., Kuzmanovich J., Zhang J.J., Shephard-Todd-Chevalley theorem for skew polynomial rings, Algebr. Represent. Theory 13 (2010), 127-158, arXiv:0806.3210. 